\title{
Comparison of Cryotop and ReproCarreir products for cryopreservation of bovine blastocysts through survival rate and blastocysts quality
}

\author{
Jin-Woo Kim ${ }^{1,2, \#, ~ S e u l-G i ~ Y a n g, ~}{ }^{1,2, \#}$, Hyo-Jin Park ${ }^{1,2}$, Ju Hwan Kim³ ${ }^{3}$ Dong-Mok Lee ${ }^{4}$, Seong-Min Woo , \\ Hyun-Jeong Kim ${ }^{1}$, Hyun Ah Kim ${ }^{1}$, Jae-Hoon Jeong ${ }^{1}$, Min Ji Lee ${ }^{1}$ and Deog-Bon Koo ${ }^{1,2, *}$ \\ ${ }^{1}$ Department of Biotechnology, College of Engineering, Daegu University, Gyeongsan 38453, Korea \\ ${ }^{2}$ Institute of Infertility, Daegu University, Gyeongsan 38453, Korea \\ ${ }^{3}$ Modu Science, Sejong 30128, Korea \\ ${ }^{4}$ Biomedical Manufacturing Technology Center, Korea Institute of Industrial Technology, Yeongcheon 38822, Korea
}

Received May 25, 2020

Accepted June 12, 2020

\section{*Correspondence}

Deog-Bon Koo

E-mail:dbkoo@daegu.ac.kr

ORCID

https://orcid.org/0000-0001-7825-9598

"These authors contributed equally to this work.

\begin{abstract}
Cryopreservation is used for blastocyst preservation of most mammalian embryos and is an important technique for breeding. We aimed to compare the efficiency of the cryopreservation method using the standard Cryotop device and the ReproCarrier device, a domestic product manufactured in Korea. The efficacy of the two devices was analyzed based on the survival rate, intracellular levels of reactive oxygen species (ROS), and apoptosis of the vitrified bovine blastocysts. The survival rates of the vitrified-warmed blastocysts were similar between the ReproCarrier group $(58.4 \pm 17.7 \%)$ and Cryotop group $(59.9 \pm 14.1 \%)$. Intracellular ROS levels and apoptotic index were determined by DCFDA staining and TUNEL assay. Changes in intracellular ROS levels, number of total nuclei, and cellular apoptosis of vitrified blastocysts after cryopreservation were not significantly different between the two groups. These results indicate that the ReproCarrier device method is as effective as the standard Cryotop method for vitrification of bovine blastocysts in vitro.
\end{abstract}

Keywords: bovine blastocyst, cryopreservation, Cryotop, ReproCarrier, vitrification

\section{INTRODUCTION}

Cryopreservation of oocytes and embryos in assisted reproductive technology for application in domestic animals and humans is very important to preserve the maternal and paternal genetic complements. Cryopreservation allows widespread use of valuable animal embryos to improve the chances of pregnancy. However, intracellular ice formation in cryopreservation methods for oocytes and embryos can lead to fatal damage. To overcome this issue of intracellular ice formation, slow freezing and vitrification methods are most widely used (Mucci et al., 2006).

The balance of redox reactions in cells is very crucial for maintaining the metabolic environment and gene expression during embryonic development. However, high levels of reactive oxygen species (ROS) were seen in preimplantation embryos of cattle (Min et al., 2014). ROS, such as hydrogen peroxide, cause oxidative stress, which is known to cause DNA damage and apoptosis (Takahashi, 2012). Therefore, regulating oxidative stress and ROS production during the vitrification-warming process is criti- 
cal for blastocyst survival as well as its quality after the process.

A previous study demonstrated the process of apoptosis of bovine blastocysts (Paschoal et al., 2017). Apoptosis is an important indicator for evaluating the quality of blastocysts. TUNEL analysis, the most used method for identifying apoptotic cells, can confirm apoptosis through the labeling of a wide range of oligonucleosomal DNA fragments produced by endogenous DNase activity during the apoptosis process. Increased percentage of apoptotic cells is also an important indicator of the quality in embryo cryopreservation (Park et al., 2006).

Currently, most of the cryopreservation-related products for slow freezing and vitrification procedures are manufactured in foreign countries (Dos Santos-Neto et al., 2017). Cryotop (Kitazato Corporation, Japan) is a representative device developed for vitrification and cryopreservation of embryos. Cryotop is associated with high survival rate and is widely used for sensitive oocytes and blastocyst stage. However, domestic production of cryopreservation-related products is still insufficient. Hence, this study aimed to compare the efficacy of the ReproCarrier (Biomedical Manufacturing technology Center, Korea), a domestic product, and the Cryotop for vitrification of bovine in vitro-produced (IVP) blastocysts by analysis of survival rate and blastocyst quality after thawing.

\section{MATERIALS AND METHODS}

\section{Chemicals}

Unless noted otherwise, all chemicals used in this study were purchased from Sigma-Aldrich Korea (Sigma-Aldrich Korea, Yongin, Korea).

\section{In vitro production of bovine embryos}

In vitro maturation (IVM) of bovine oocytes was performed as described by Min et al. (2014) with slight modification. Briefly, bovine ovaries were acquired from a local slaughterhouse and transported to the laboratory in $0.9 \%$ saline containing $75 \mu \mathrm{g} / \mathrm{mL}$ penicillin $\mathrm{G}$ sodium salt at $30-35^{\circ} \mathrm{C}$. Cumulus-oocyte complexes (COCs) were aspirated from follicles (3-6 $\mathrm{mm}$ in diameter) using a disposable $10-\mathrm{mL}$ syringe with an 18-gauge needle. Aspirated COCs with at least three layers of compact cumulus cells and homogeneous cytoplasm were collected using mouth pipetting, then washed thrice in Tyrode's lactate- $N$ 2hydroxyethlpiperazine-ethanesulfonic acid (TL-HEPES). Approximately 10-15 oocytes were matured in $50 \mu \mathrm{L}$ of IVM medium in a 60-mm dish (Nunc, Roskilde, Denmark) under paraffin oil (Junsei, Tokyo, Japan) for 20-22 h at $38.5^{\circ} \mathrm{C}$ under an atmosphere of $5 \% \mathrm{CO}_{2}$ in air. The medium used for oocyte maturation was TCM-199 (GibcoBRL, Grand Island, NY, USA) supplemented with $0.2 \mathrm{mM}$ $\mathrm{Na}$-pyruvate, $0.6 \mathrm{mM}$ cysteine, $10 \mathrm{IU} / \mathrm{mL}$ pregnant mare's serum gonadotropin, $10 \mathrm{IU} / \mathrm{mL}$ human chorionic gonadotropin, $25 \mu \mathrm{M} \beta$-mercaptoethanol, $10 \mathrm{ng} / \mathrm{mL}$ epidermal growth factor, $25 \mu \mathrm{g} / \mathrm{mL}$ gentamycin, 10\% FBS (GibcoBRL) and $1 \mu \mathrm{g} / \mathrm{mL}$ estradiol- $\beta$. After IVM, 15 oocytes were fertilized with frozen-thawed sperm at a concentration of $2 \times 10^{6}$ cells $/ \mathrm{mL}$ in $50 \mu \mathrm{L}$ of fertilization medium. The fertilization medium consisted of Fert-TALP medium supplemented with $0.2 \mathrm{mM} \mathrm{Na-pyruvate,} 25 \mu \mathrm{g} / \mathrm{mL}$ gentamycin, and $0.6 \%$ BSA. In addition, $10 \mu \mathrm{g} / \mathrm{mL}$ heparin, $80 \mu \mathrm{M}$ penicillamine, $4 \mu \mathrm{M}$ hypo-taurine, and $2 \mu \mathrm{M}$ epinephrine were also added to the fertilization drops. After $22 \mathrm{~h}$ of insemination, cumulus-enclosed oocytes were stripped using gentle pipetting and transferred to CR1-aa medium containing $0.4 \mathrm{mM}$ Na-pyruvate, $1 \mathrm{mM}$ glutamine, 0.3 $\mathrm{mg} / \mathrm{mL}$ glutathione, $25 \mu \mathrm{g} / \mathrm{mL}$ gentamycin, and $0.3 \%$ BSA for in vitro culture (IVC). After culture for two days, the cleaved embryos were further cultured in medium containing $50 \mu \mathrm{L}$ of CR1-aa with $0.4 \mathrm{mM} \mathrm{Na-pyruvate,} 1 \mathrm{mM}$ glutamine, $0.3 \mathrm{mg} / \mathrm{mL}$ glutathione, $25 \mu \mathrm{g} / \mathrm{mL}$ gentamycin, and $10 \% \mathrm{FBS}$ for four days at $38.5^{\circ} \mathrm{C}$ in $5 \% \mathrm{CO}_{2}$ in air.

\section{Vitrification and warming procedure}

As one experimental method, cryopreservation was carried out by vitrification with a Cryotop (Kitazato Supply Co, Fujinomiya, Japan, Fig. 1A) using a slightly modified version of the procedure described by Moulavi (2019). Briefly, one or two blastocysts were transferred into equilibration solution (ES) consisting of $7.5 \%$ dimethyl sulfoxide (DMSO) and 7.5\% ethylene glycol (EG) in PBS supplemented with $20 \%$ FBS at room temperature for 5 min. Next, blastocysts were transferred into vitrification solution (VS) consisting of 15\% DMSO, 15\% EG, and 0.5 M sucrose dissolved in PBS containing 20\% FBS. After 40$45 \mathrm{~s}$, the blastocysts were loaded into a Cryotop and sank into liquid nitrogen. The process from exposure to VS to sinking into liquid nitrogen was completed within $1 \mathrm{~min}$ at room temperature. Vitrified blastocysts were warmed 
A

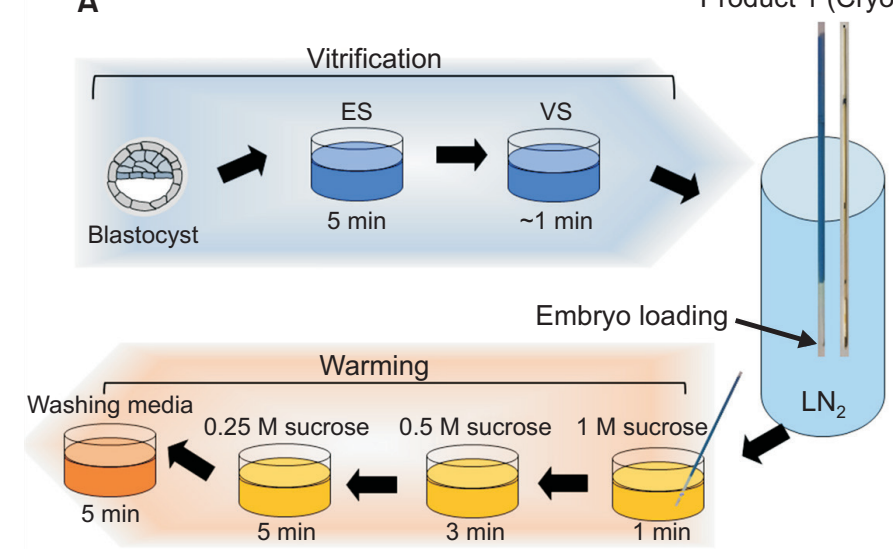

Product 2

(ReproCarrier)

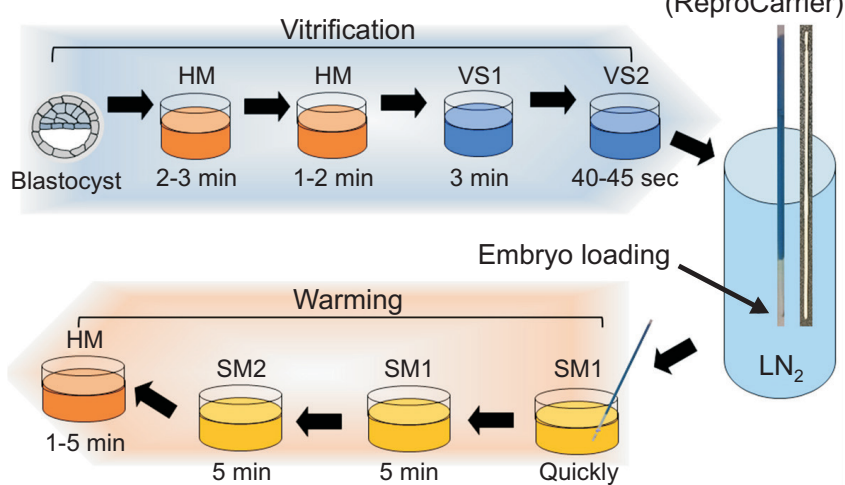

Fig. 1. Experimental diagram of the vitrification methods of bovine blastocysts using Cryotop and ReproCarrier products. (A) Description of vitrification protocol using Cryotop. (B) Graphical description of vitrification experimental methods using ReproCarrier.

by immersing the Cryotop directly into warming solution (1.0 M sucrose in PBS with 20\% FBS) for $1 \mathrm{~min}$, after which they were transferred to dilution solution $(0.5 \mathrm{M}$ sucrose in PBS with 20\% FBS) for 3 min, and then to dilution solution ( $0.25 \mathrm{M}$ sucrose in PBS with $20 \% \mathrm{FBS}$ ) for $5 \mathrm{~min}$ at room temperature. Subsequently, blastocysts were incubated for $5 \mathrm{~min}$ in washing solution (PBS with $20 \%$ FBS) at room temperature. As another experimental method, the vitrification process was carried out using the ReproCarrier (Fig. 1B). Briefly, one or two blastocysts were transferred into handling medium (HM; Modu Science, Korea) at room temperature for 2-3 min, followed by a second transfer to $\mathrm{HM}$ at room temperature for 1-2 min. Next, blastocysts were transferred into vitrification solution 1 (VS1) at room temperature for $3 \mathrm{~min}$, followed by transfer into vitrification solution 2 (VS2) at room temperature for 40-45 s. Thereafter, the blastocysts were loaded into a ReproCarrier and sunk into liquid nitrogen. The process from exposure to VS2 to sinking into liquid nitrogen was completed within 1 min. Vitrified blastocysts were warmed by immersing the ReproCarrier directly into sucrose medium 1 (SM1) solution, followed by immediate transfer to the next SM1 solution at room temperature for 5 min. Next, blastocysts were transferred into sucrose medium 2 (SM2) solution for 5 min. Finally, blastocysts were transferred into HM. Survival of vitrified-warmed blastocysts was determined according to re-expansion rates after $24 \mathrm{~h}$ of recovery in culture medium.

\section{Measurement of ROS levels}

The level of ROS in each embryo was measured using the difluorodihydrofluorescein diacetate method (H2DCFDA; Molecular Probes, Eugene, OR, USA) described previously (Choi et al., 2008). Vitrified-warmed blastocysts in IVC medium were washed thrice with $0.1 \%$ polyvinylalcohol (PVA) in PBS. Blastocysts were transferred into IVC medium containing $5 \mu \mathrm{M} \mathrm{H}_{2} \mathrm{DCFDA}$ for $30 \mathrm{~min}$ at $38.5^{\circ} \mathrm{C}$. The intensity of H2DCFDA was measured with an iRiS Digital Cell Imaging System (Logos Biosystems, Gyeonggido, South Korea). The measured fluorescence images were analyzed by Image J software Version 1.38 (National Institutes of Health, Bethesda, MD, USA).

\section{Assessment of apoptosis in blastocysts}

Apoptotic cells in vitrified-warmed blastocysts were detected using an In Situ Cell Death Detection kit (Roche Diagnostics GmbH, Mannheim, Germany) according to the manufacturer's instructions. On day 7 , blastocysts were washed with $0.1 \%$ PVA in PBS and then fixed in $3.7 \%$ formaldehyde in PBS for $1 \mathrm{~h}$ at room temperature. Next, blastocysts were permeabilized using $0.5 \%$ Triton X-100 for 30 min at room temperature. The fixed blastocysts were incubated in TUNEL reaction medium for $1 \mathrm{~h}$ at $38.5^{\circ} \mathrm{C}$, then washed and mounted on slide glass with DAPI solution. Stained blastocysts were examined under an iRiS Digital Cell Imaging System (Logos Biosystems, Gyeonggi-do, South Korea).

\section{Statistical analysis}

All percentage data obtained in the present study are presented as the mean \pm SD. All data were analyzed using student's $t$-tests and plotted using the GraphPad Prism 5.0 
software package (San Diego, CA, USA). Histogram values of densitometry were measured by ImageJ software (NIH, USA).

\section{RESULTS}

\section{Comparison of the instructions and features of the Cryotop and ReproCarrier products}

Cryotop is composed of "Diamond tip", "Winder sheet", "Conjunction reshaped", "Widened body", and "Identification marks" (Fig. 2A). The length and weight of Cryotop were measured to be $13 \mathrm{~cm}$ and $0.62 \mathrm{~g}$, respectively. Blastocysts mounted wider sheet as flat part (Fig. 2C). The lid of the Cryotop was closed in the liquid nitrogen. The ReproCarrier consists of "Embryo loading well", "Gas passage way", and "Identification logo" (Fig 2B). The length and weight of ReproCarrier were measured to be $13.2 \mathrm{~cm}$ and $1.11 \mathrm{~g}$, respectively. The blastocysts were mounted into the embryo-loading well (Fig. 2D). The lid of the ReproCarrier was closed before placing it in the liquid nitrogen. Notably, the ReproCarrier has a distinct site as the loading well to place the embryos as compared to the Cryotop.

Comparison of survival rate, intracellular ROS, and apoptosis level in the vitrified-warmed bovine blastocysts using Cryotop and ReproCarrier

To investigate the efficacy of the Cryotop and ReproCarrier products in survival of the vitrified-warmed bovine blastocysts after their recovery, we evaluated the morphology of normal blastocysts using a microscope (Leica, Solms, Germany) (Fig. 3A). Survival rates of the vitrified-warmed blastocysts were not significantly different between the Cryotop (59.9 $\pm 14.1 \%$ ) group and ReproCarrier (58.4 $\pm 17.7 \%$ ) group (Fig. 3B). Based on this result, both methods were verified to have similar effects on blastocyst survival after cryopreservation.

To confirm the damage caused to the bovine blastocyst by cryopreservation, we measured intracellular ROS levels by H2DCFDA staining of vitrified-warmed bovine blasto-
A

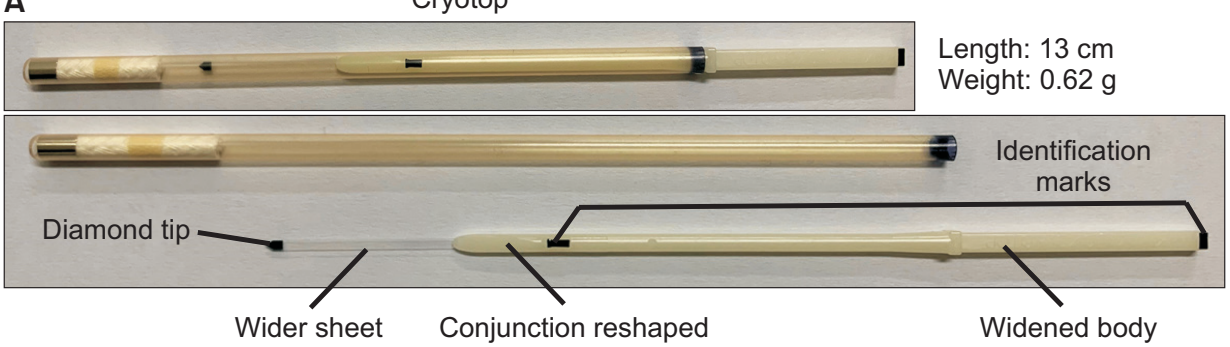

B
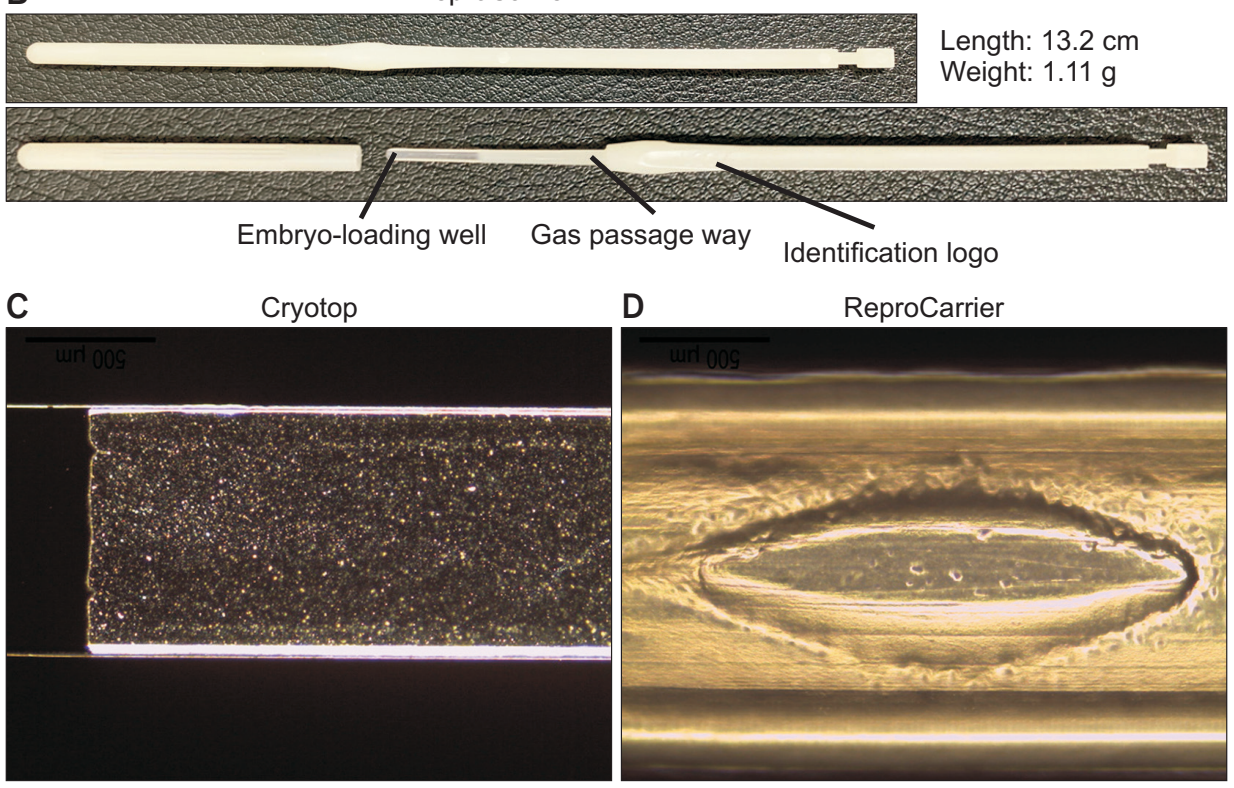

D

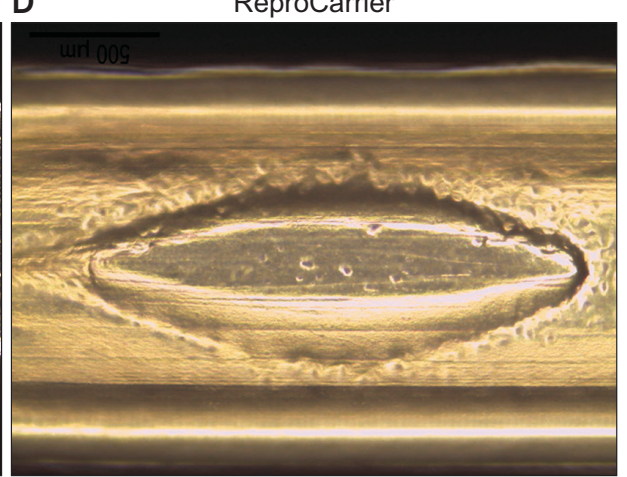

Fig. 2. Comparison of standard features for vitrification products. (A) Length, weight, and standard features of Cryotop. (B) Length, weight, and standard features of ReproCarrier. (C) Bovine blastocysts were mounted flat site on Cryotop. (D) Bovine blastocysts were mounted in the embryoloading well on the ReproCarrier. 
A

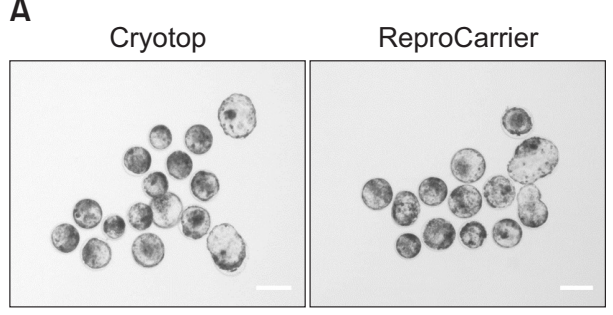

B

\begin{tabular}{ccc}
\hline Type & $\begin{array}{c}\text { No. of } \\
\text { blastocysts } \\
\text { thawed }\end{array}$ & $\begin{array}{c}\text { No. of surviving } \\
\text { blastocysts (\%) }\end{array}$ \\
\hline Cryotop & 28 & $16(59.9 \pm 14.1)$ \\
ReproCarrier & 36 & $19(58.4 \pm 17.7)$ \\
\hline
\end{tabular}
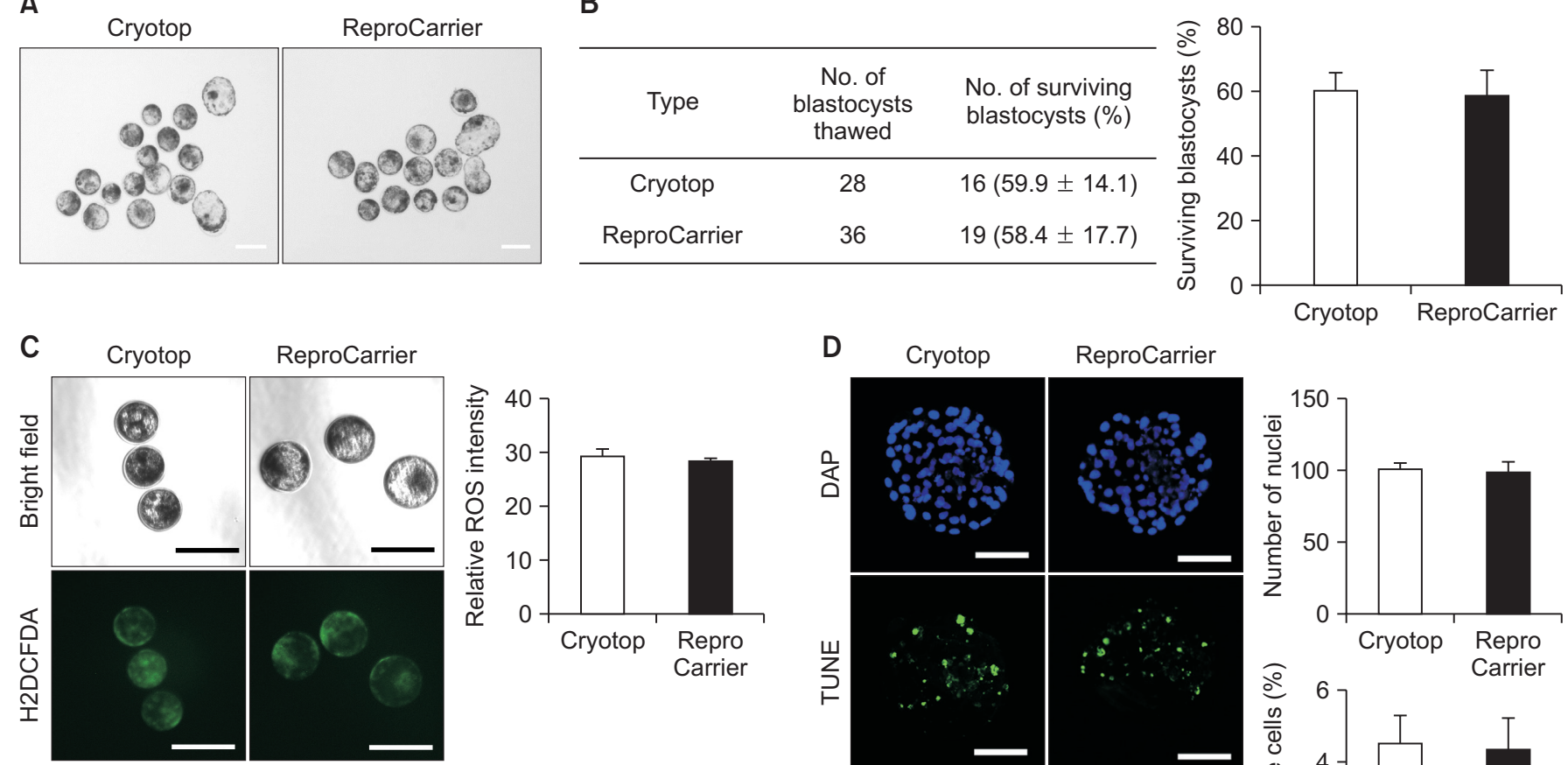

D

D Cryotop ReproCarrier
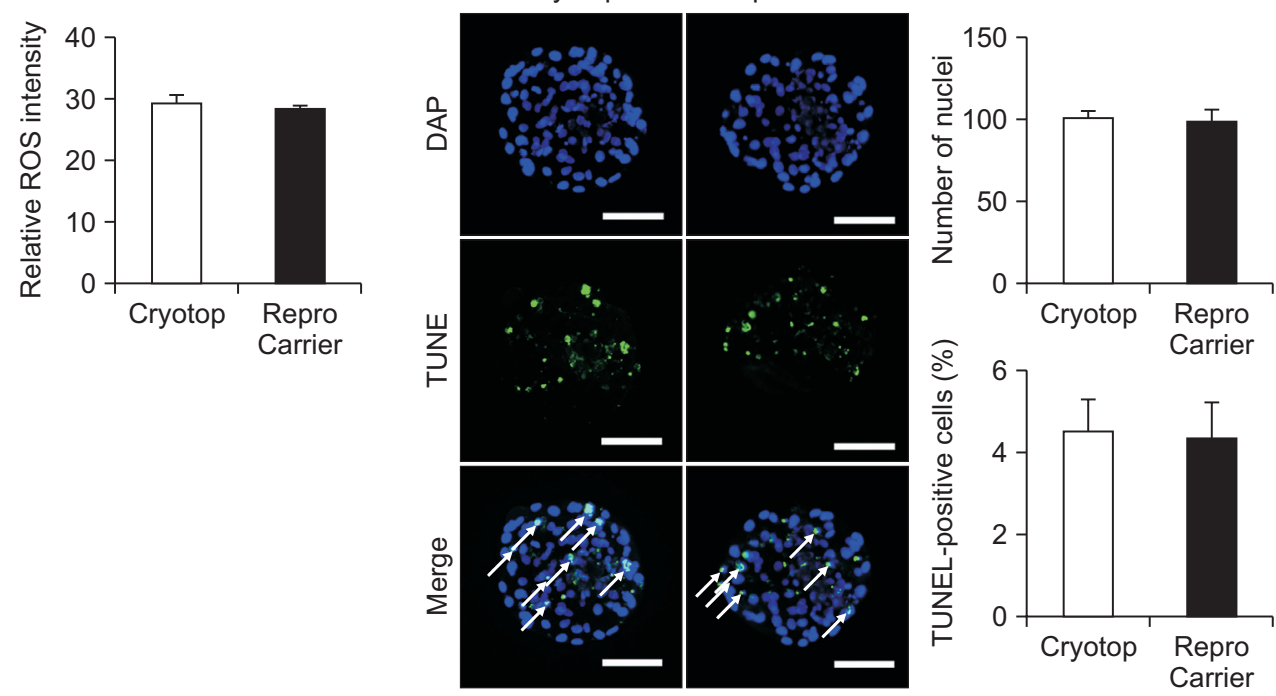

Fig. 3. Comparison of survival rate, intracellular ROS production, and apoptosis level in vitrified-warmed bovine blastocysts obtained using Cryotop and ReproCarrier products. (A) Morphology of vitrified-warmed bovine blastocysts visualized using microscope. (B) The survival rates of vitrified-warmed bovine blastocysts using Cryotop and ReproCarrier. (C) Vitrified-warmed bovine blastocysts using Cryotop and ReproCarrier were evaluated for intracellular ROS (green) by H2DCFDA staining. Scale bar $=100 \mu \mathrm{m}$. Data in the bar graph represent means \pm SD from three independent experiments. (D) Colocalization of DAPI and TUNEL stains at the blastocyst stage. The blastocysts were stained for chromosome (blue) and fragmented DNA (green). The white arrows indicate a merged spot with chromosome and fragmented DNA. Data in the bar graph represent the means \pm SD from three independent experiments. Scale bar $=200 \mu \mathrm{m}$.

cysts (Fig. 3C). The intracellular ROS intensity in blastocysts subjected to Cryotop method showed similar results to that in blastocysts subjected to the ReproCarrier. To investigate fragmented DNA, we performed DAPI and TUNEL staining of vitrified-warmed bovine blastocysts (Fig. 3D). The number of nuclei were similar in the blastocysts of both Cryotop (105.3 \pm 12.3$)$ and ReproCarrier (100.5 \pm 17.5) groups. In addition, the number of TUNEL-positive cells using Cryotop $(4.5 \pm 2.5 \%)$ and ReproCarrier ( $4.8 \pm$ $2.9 \%)$ were not significantly different. Thus, cryopreservation-induced damages such as ROS production and fragmented DNA were not significantly different between the Cryotop and ReproCarrier groups.

\section{DISCUSSION}

In the present study, we confirmed the similar survival rates of vitrified-warmed bovine blastocysts prepared using the Cryotop and ReproCarrier products. In addition, blastocysts obtained from the ReproCarrier method showed a similar level of cellular apoptosis due to cryodamage as observed in the Cryotop group. Thus, our results demonstrated the similar efficacy of the ReproCarrier device as a domestic product in comparison to the Cryotop device for vitrification of bovine blastocyst.

Vitrification is a commonly used simple cryopreservation method yielding high survival rates. Cryotop method is the most representative of the vitrification cryopreservation method. Currently, domestic products related to 
cryopreservation, such as ReproCarrier are rare. ReproCarrier is a cryopreservation product developed in Korea (Fig. 2B and Fig. 2D). Therefore, we confirmed whether ReproCarrier is suitable for cryopreservation by comparing the survival rate of blastocysts as well as the cryo-damage in vitrified-warmed blastocysts obtained through ReproCarrier or Cryotop products. No difference was found in the viability and quality of blastocysts obtained by the two methods, as evident from the evaluation of ROS production and apoptosis (Fig. 3C, 3D). ROS is known to play a variety of roles during the respiratory process of cells. However, ROS imbalance is known to cause DNA damage, endoplasmic reticulum (ER)-stress (He et al., 2018), and apoptosis in mammalian cells (Ansari et al., 2018). In addition, increased ROS during embryo development has been associated with decreased blastocyst formation (Yang et al., 2018). In other words, regulating ROS production is important to improve blastocyst competence (Moulavi et al., 2019) and survival of vitrified blastocysts (Pereira et al., 2019). During cryopreservation, blastocysts can be damaged by various factors such as membrane, DNA, and/or thermal damage (Inaba et al., 2016). A previous study indicated that endogenous ROSmediated cellular cryo-damage is related to blastocyst formation rate and survival (Lee et al., 2019). In the current study, we observed that the intracellular ROS production was not significantly different in the ReproCarrier and Cryotop methods.

During IVP progression of bovine embryos, apoptosis is an important indicator of the quality of blastocysts, and an increase in the number of apoptotic cells indicates that the in vitro culture environment is inadequate (Nánássy et al., 2008). The TUNEL assay in our study revealed that the total cell number and apoptotic cells were not significantly different in the ReproCarrier and Cryotop groups (Fig. 3D).

\section{CONCLUSION}

In conclusion, the results of the present study suggest that vitrified warmed bovine blastocyst obtained using the ReproCarrier method is not significantly different from that obtained using the Cryotop in terms of survival rate of blastocyst formation, number of apoptotic cells, and intracellular ROS levels. Therefore, we suggest that ReproCarrier has similar efficiency to the Cryotop for cryopreservation of bovine blastocysts.

\section{CONFLICTS OF INTEREST}

No potential conflict of interest relevant to this article was reported.

\section{ACKNOWLEDGEMENTS}

This work was supported by X-mind Corps program of National Research Foundation of Korea (NRF) funded by the Ministry of Science, ICT (2019H1D8A110986511).

\section{AUTHOR CONTRIBUTIONS}

Conceptualization: Jin-Woo Kim, Seul-Gi Yang and Deog-Bon Koo

Data curation: Jin-Woo Kim and Seul-Gi Yang

Formal analysis: Jin-Woo Kim, Seul-Gi Yang, Seong-Min

Woo, Hyun-Jeong Kim, Hyun Ah Kim, Jae-Hoon Jeong and Min Ji Lee

Funding acquisition: Deog-Bon Koo

Investigation: Jin-Woo Kim and Seul-Gi Yang

Methodology: Jin-Woo Kim, Seul-Gi Yang and Ju Hwan Kim

Project administration: Jin-Woo Kim, Seul-Gi Yang and Deog-Bon Koo

Resources: Dong-Mok Lee, Ju Hwan Kim and Deog-Bon Koo Supervision: Deog-Bon Koo

Validation: Jin-Woo Kim and Seul-Gi Yang

Writing - original draft: Jin-Woo Kim and Seul-Gi Yang

Writing - review \& editing: Seul-Gi Yang, Hyo-Jin Park and Deog-Bon Koo

\section{AUTHOR'S POSITION AND ORCID NO.}

JW Kim, PhD, https://orcid.org/0000-0002-0089-680X

SG Yang, PhD Candidate, https://:orcid.org/0000-0003-3247-2443

HJ Park, PhD, https://orcid.org/0000-0003-3287-2914

JH Kim, PhD Candidate, https://orcid.org/0000-0002-8116-8141

DM Lee, PhD, https://orcid.org/0000-0002-2423-3322

SM Woo, BS Candidate, https://orcid.org/0000-0003-2677-4461

HJ Kim, BS Candidate, https://orcid.org/0000-0001-6017-5177 
HA Kim, BS Candidate, https://orcid.org/0000-0002-9215-7640

JH Jeong, BS Candidate, https://orcid.org/0000-0002-0202-4765

MJ Lee, BS Candidate,

https://orcid.org/0000-0003-1186-8975

DB Koo, Professor,

https://orcid.org/0000-0001-7825-9598

\section{REFERENCES}

Ansari SM, Saquib Q, Attia SM, Abdel-Salam EM, Alwathnani HA, Faisal M, Alatar AA, Al-Khedhairy AA, Musarrat J. 2018. Pendimethalin induces oxidative stress, DNA damage, and mitochondrial dysfunction to trigger apoptosis in human lymphocytes and rat bone-marrow cells. Histochem. Cell Biol. 149:127-141.

Choi J, Park SM, Lee E, Kim JH, Jeong YI, Lee JY, Park SW, Kim HS, Hossein MS, Jeong YW, Kim S, Hyun SH, Hwang WS. 2008. Anti-apoptotic effect of melatonin on preimplantation development of porcine parthenogenetic embryos. Mol. Reprod. Dev. 75:1127-1135.

Dos Santos-Neto PC, Cuadro F, Barrera N, Crispo M, Menchaca A. 2017. Embryo survival and birth rate after minimum volume vitrification or slow freezing of in vivo and in vitro produced ovine embryos. Cryobiology 78:8-14.

He Q, Zhou X, Liu Y, Gou W, Cui J, Li Z, Wu Y, Zuo D. 2018. Titanium dioxide nanoparticles induce mouse hippocampal neuron apoptosis via oxidative stress- and calcium imbalance-mediated endoplasmic reticulum stress. Environ. Toxicol. Pharmacol. 63:6-15.

Inaba Y, Miyashita S, Somfai T, Geshi M, Matoba S, Dochi O, Nagai T. 2016. Cryopreservation method affects DNA fragmentation in trophectoderm and the speed of re-expansion in bovine blastocysts. Cryobiology 72:86-92.

Lee AR, Hong K, Choi SH, Park C, Park JK, Lee JI, Bang JI, Seol DW, Lee JE, Lee DR. 2019. Anti-apoptotic regulation contributes to the successful nuclear reprogramming using cryopreserved oocytes. Stem Cell Reports 12:545-556.
Min SH, Song BS, Yeon JY, Kim JW, Bae JH, Park SY, Lee YH, Chang KT, Koo DB. 2014. A cathepsin B inhibitor, E-64, improves the preimplantation development of bovine somatic cell nuclear transfer embryos. J. Reprod. Dev. 60:21-27.

Moulavi F, Soto-Rodriguez S, Kuwayama M, Asadi-Moghaddam B, Hosseini SM. 2019. Survival, re-expansion, and pregnancy outcome following vitrification of dromedary camel cloned blastocysts: a possible role of vitrification in improving clone pregnancy rate by weeding out poor competent embryos. Cryobiology 90:75-82.

Mucci N, Aller J, Kaiser GG, Hozbor F, Cabodevila J, Alberio RH. 2006. Effect of estrous cow serum during bovine embryo culture on blastocyst development and cryotolerance after slow freezing or vitrification. Theriogenology 65:1551-1562.

Nánássy L, Lee K, Jávor A, Macháty Z. 2008. Effects of activation methods and culture conditions on development of parthenogenetic porcine embryos. Anim. Reprod. Sci. 104:264-274.

Park SY, Kim EY, Cui XS, Tae JC, Lee WD, Kim NH, Park SP, Lim JH. 2006. Increase in DNA fragmentation and apoptosis-related gene expression in frozen-thawed bovine blastocysts. Zygote 14:125-131.

Paschoal DM, Sudano MJ, Schwarz KRL, Maziero RRD, Guastali MD, Crocomo LF, Magalhães LCO, Martins Jr. A, Leal CLV, Landim-Alvarenga FDC. 2017. Cell apoptosis and lipid content of in vitro-produced, vitrified bovine embryos treated with forskolin. Theriogenology 87:108-114.

Pereira BA, Zangeronimo MG, Castillo-Martín M, Gadani B, Chaves BR, Rodríguez-Gil JE, Bonet S, Yeste M. 2019. Supplementing maturation medium with insulin growth factor I and vitrification-warming solutions with reduced glutathione enhances survival rates and development ability of in vitro matured vitrified-warmed pig oocytes. Front. Physiol. 9:1894.

Takahashi M. 2012. Oxidative stress and redox regulation on in vitro development of mammalian embryos. J. Reprod. Dev. 58:1-9.

Yang SG, Park HJ, Kim JW, Jung JM, Kim MJ, Jegal HG, Kim IS, Kang MJ, Wee G, Yang HY, Lee YH, Seo JH, Kim SU, Koo DB. 2018. Mito-TEMPO improves development competence by reducing superoxide in preimplantation porcine embryos. Sci. Rep. 8:10130. 